\title{
Evidence of charge carrier number fluctuations in InN thin films?
}

\author{
Geeta Rani Mutta ${ }^{* \dagger}$, Bruno Guillet ${ }^{\dagger}$, Laurence Méchin ${ }^{\dagger}$, Arantxa Vilalta-Clemente ${ }^{\dagger}$, Javier Grandal ${ }^{\ddagger}$, \\ Miguel A. Sánchez-García ${ }^{\ddagger}$, Sara Martin ${ }^{\ddagger}$, Fernando Calle ${ }^{\ddagger}$, \\ Pierre Ruterana* and Jean-Marc Routoure ${ }^{\dagger}$ \\ * CIMAP UMR 6252 CNRS-ENSICAEN-CEA-UCBN, \\ 6, Boulevard du Maréchal Juin, 14050 Caen Cedex, France \\ † GREYC, UMR CNRS 6072, ENSICAEN, Université de Caen Basse-Normandie, \\ 6 Boulevard du maréchal Juin, 14050 Caen Cedex, France \\ contact:jean-marc.routoure@unicaen.fr \\ $\ddagger$ ISOM and Dept. de Ingeniería Electrónica, E.T.S.I. Telecomunicación, Universidad Politécnica de Madrid, \\ Ciudad Universitaria, 28040 Madrid, Spain
}

\begin{abstract}
Due to its small band-gap and its high mobility, InN is a promising material for a large number of key applications like band-gap engineering for high efficiency solar cells, light emitting diodes, and high speed devices. Unfortunately, it has been reported that this material exhibits strong surface charge accumulation which may depend on the type of surface. Current investigations are conducted in order to explain the mechanisms which govern such a behavior and to look for ways of avoiding it and/or finding applications that may use such an effect. In this framework, low frequency noise measurements have been performed at different temperatures on patterned MBE grown InN layers. The evolution of the $1 / f$ noise level with temperature in the $77 \mathrm{~K}-300 \mathrm{~K}$ range is consistent with carrier number fluctuations thus indicating surface mechanisms: the surface charge accumulation is confirmed by the noise measurements.
\end{abstract}

\section{INTRODUCTION}

During the last decade Indium Nitride ( $I n N)$ has been the subject of intense research which led to the redefinition of its band gap from 1.89 to $0.69 \mathrm{eV}$ and investigations of its many interesting properties like electron effective mass [1], [2], the largest mobility [3], the highest peak and saturation electron drift velocities [2]. This has made InN a promising candidate for high speed electronic devices and optoelectronic applications up to terahertz frequency range [4], [5]. Despite these novel properties of $\mathrm{InN}$, there are still many challenges for device fabrication which are related to material's quality, but also to the presence of an intrinsic charge accumulation at surfaces [6].

In this paper, low frequency noise measurements performed at different temperatures from $100 \mathrm{~K}$ up to $300 \mathrm{~K}$ on MBE grown InN thin films are shown and discussed. First experimental conditions and samples are presented. Results are then shown and a model proposed to explain the evolution of the $1 / \mathrm{f}$ noise level with the temperature is then given. Finally, some conclusions are proposed.

\section{EXPERIMENTAL CONDITIONS}

The studied InN films were grown on a $5 \mu \mathrm{m}$ thick GaN template on top of a $300 \mu \mathrm{m}$ thick $\mathrm{Al}_{2} \mathrm{O}_{3}$ substrate. The thickness $\mathrm{Z}$ of the InN layer was $345 \mathrm{~nm}$. For electrical measurements versus temperature, we used classical Transmission Line Method (TLM) structures : the size of the pads was $94 \times 48 \mu m^{2}$ and the distances between the metallic pads were $38 \mu \mathrm{m}, 57 \mu \mathrm{m}, 114 \mu \mathrm{m}, 170 \mu \mathrm{m}$ and $210 \mu \mathrm{m}$. The width was $\mathrm{W}=94 \mu \mathrm{m}$. The same structure has been reproduced over the wafer. A photograph of the sample is given in figure 1 . Using the whole range of length in the TLM structures, it has been checked that the contact resistances were negligible at all the temperatures.

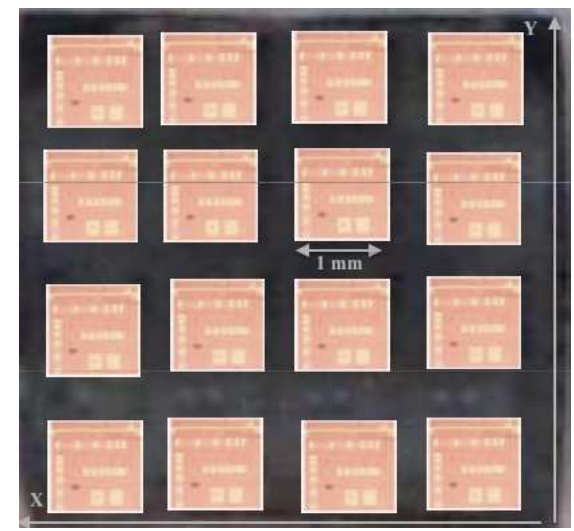

Fig. 1. Photograph of the sample used for the mapping and the temperature studies.

DC and noise measurements were performed only with the $\mathrm{L}=210 \mu \mathrm{m}$ length sample at different bias current for each temperature. A four probe configuration technique was used with a home-made read-out set-up. Due to the high output impedance and low noise level of the 
DC current source used, the current contact noise can be neglected [7]. The electrical resistivity of the samples were extracted from the DC measurement and finally the voltage noise spectral density $S_{V}$ was estimated from 10 $\mathrm{Hz}$ up to $100 \mathrm{kHz}$ using an HP3562A spectrum analyzer.

\section{Results}

First a mapping of the noise level as well as the electrical resistivity was performed at room temperature. Each position was referenced by the $(\mathrm{X}, \mathrm{Y})$ position on the sample as indicated in figure 1. Results for electrical resistivity and 1 /f noise level at $1 \mathrm{~Hz}, 1 \mathrm{~V}$ (defined as $\mathrm{K}$ in equation 1) are given in the figure 2 .
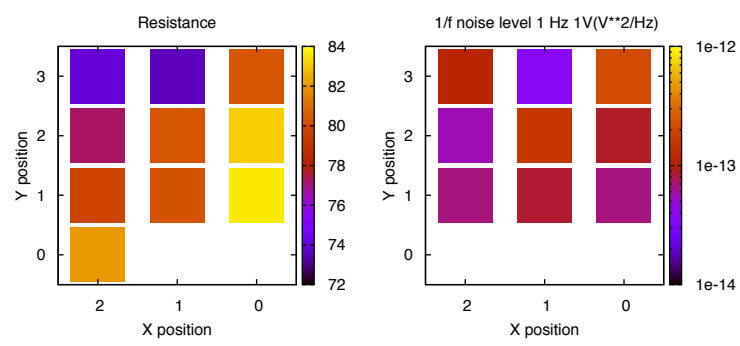

Fig. 2. Result of the "mapping" done at room temperature. No clear dependency with the position on the sample, the electrical resistivity and $1 /$ f noise level at $1 \mathrm{~Hz}$ was found

From this figure, no clear dependency between the position and the electrical resistivity and the $1 / \mathrm{f}$ noise at $1 \mathrm{~Hz} 1 \mathrm{~V}$ was found. Due to the small dispersions in the values for electrical resistivity and even noise level (less than two orders of magnitude in the $1 / \mathrm{f}$ noise level dispersion is not a important value 1 /f noise !), it indicates a good homogeneity of the film as well as a good level of confidence in the film growth deposition. In the following, the sample X1Y1 which exhibits a "mean" behavior has been chosen for the temperature investigation.

At each temperature, it was found that the electrical resistivity $\rho$ does not depend on the bias current showing both a good ohmic contact as well as no self heating effect. It was also found that $S_{V}$ consists mainly of a white noise due to the thermal noise and $1 /$ f noise figure 3 . Some lorentzian contributions has been observed at low temperature but it will not be discussed here. The $1 / \mathrm{f}$ noise level has a quadratic dependence with the voltage across the sample [8]. Finally, one can write for the $1 / \mathrm{f}$ noise:

$$
S_{V 1 / f}=\frac{K \cdot V^{2}}{f}
$$

A clear increase in the $1 / \mathrm{f}$ noise level $\mathrm{K}$ with the temperature has been observed (factor between 10 and 100). In the next section, a description of the model used to explain this increase is presented.

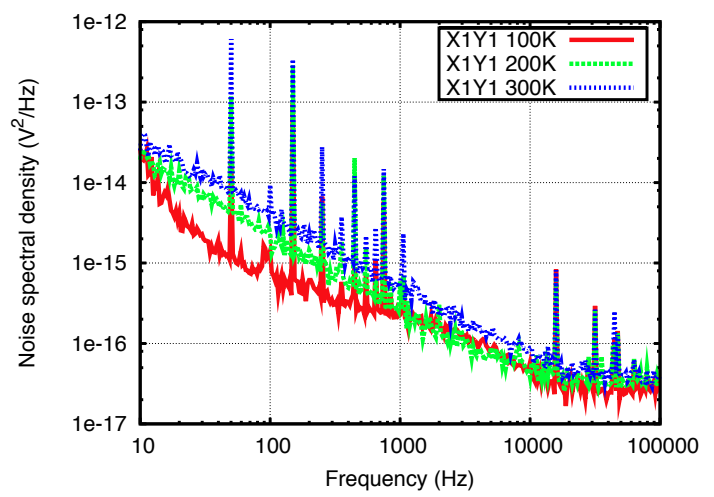

Fig. 3. Noise spectral density for the same DC current $(\mathrm{I}=10 \mathrm{~mA})$ and for 3 different temperatures $100 \mathrm{~K}, 200 \mathrm{~K}$ and $300 \mathrm{~K}$. Noise spectral density mainly consists in $1 / \mathrm{f}$ noise and white noise and $1 / \mathrm{f}$ noise level increases when the temperature increases.

\section{MOdEL DESCRIPTION AND DISCUSSION}

\section{A. Background}

It is well known that in homogeneous sample, the electrical conductivity $\sigma$ is given by $\sigma=n \cdot q \cdot \mu$ with $\mathrm{q}=1.6 \cdot 10^{-19} C, n$ the free carrier concentration and $\mu$ the free carrier mobility. Thus, $1 / \mathrm{f}$ current noise fluctuations can arise from either fluctuations in the carrier mobility $(\Delta \mu$ model $)$ or fluctuations in the carrier number $(\Delta N$ model). It follows that the voltage fluctuation spectral density depends on either mobility fluctuation spectral density $S_{\mu}$ or number fluctuation spectral density $S_{N}$ respectively given by :

$$
\frac{S_{V}}{V^{2}}=\frac{S_{\mu}}{\mu^{2}} \text { or } \frac{S_{V}}{V^{2}}=\frac{S_{N}}{N^{2}}
$$

with $N$ the total number of carriers in the sample which can be linked to the carrier concentration since $N=n$. $W \cdot L \cdot Z$.

In the following, the two model of $1 / \mathrm{f}$ noise are compared and the evolution of the $1 / \mathrm{f}$ noise level with temperature is predicted using $K=f \cdot S_{V 1 / f} / V^{2}$

\section{B. Mobility fluctuations}

Following the semi empirical approach proposed by Hooge fifty years ago [9], one can write:

$$
\frac{S_{\mu}}{\mu^{2}}=\frac{\alpha}{f \cdot N}
$$

with $\alpha$ a constant which may depend on the mobility if one assumes that only "lattice scattering causes $1 /$ f noise" [10]. 
In the present context, it is also assumed that the total number of carriers is constant over the whole range of temperature. It follows that $\mathrm{K}(300 \mathrm{~K}) / \mathrm{K}(\mathrm{T})$ can be modeled using the electrical resistance measurements with the temperature:

$$
\frac{K(T)}{K(300 K)}=\left(\frac{\mu(T)}{\mu(300 K)}\right)^{2}=\left(\frac{R(300 K)}{R(T)}\right)^{2}
$$

\section{Number fluctuations}

It is well known that generation-recombination fluctuations arising from one trap of energy level $E$ in the bandgap with a time constant $\tau$ give a spectral density given by [11]:

$$
S_{N}=\int_{E} \int_{\tau} \frac{4 \cdot \tau \cdot N_{t}(E) f_{t}(E)\left(1-f_{t}(E)\right)}{1+(2 \cdot \pi \cdot f \cdot \tau)^{2}} d E \cdot d \tau
$$

In this equation, $N_{t}$ is the trap density and $f_{t}(E)$ is the Fermi distribution that depends on the Fermi level $E_{f}$ given by $f_{t}(E)=1 /\left(1+\exp \left(\frac{E-E_{f}}{k_{B} \cdot T}\right)\right)$ with $k_{B}$ the boltzmann constant. Assuming that the trap density is constant over the band-gap (thus taking a surface origin for the traps), that the time constants are equally spaced between $\tau_{1}$ and $\tau_{2}$ and that the total number of carriers does not depend on the temperature, one can finally predict that $\mathrm{K}(300 \mathrm{~K}) / \mathrm{K}(\mathrm{T})$ can be expressed by:

$$
\frac{K(T)}{K(300 K)}=\left(\frac{T}{300}\right)
$$

\section{Discussion}

The results for the two models are plotted in figure 4 as well as some experimental data. Even if the proposed model is not able to fit in a consistent manner all the experimental measurements, it can be proposed that $1 / \mathrm{f}$ noise in our MBE grown thin films arises from number fluctuations. Only the number fluctuations model due to the surface could predict an increase of the $1 / \mathrm{f}$ noise level with the temperature. The differences between the proposed model and the measurements may come from the numbers of simplified assumptions : constant carriers concentration over the temperature range ; constant trap density of the band-gap, etc. A more confident model is under progress.

\section{Conclusions}

In this paper, it has been shown that in MBE grown InN films, no clear dependency between the position on the sample, the electrical resistivity and the $1 /$ f noise level at $1 \mathrm{~Hz}$ has been observed at room temperature thus showing a good reliability in the process technology. From the temperature study in the $100 \mathrm{~K}-300 \mathrm{~K}$ range, it has also been shown that only the surface origin of the noise due to number fluctuations could explain the increase of the noise level with the temperature. This results is coherent with the surface charge accumulation already mentioned by other studies.

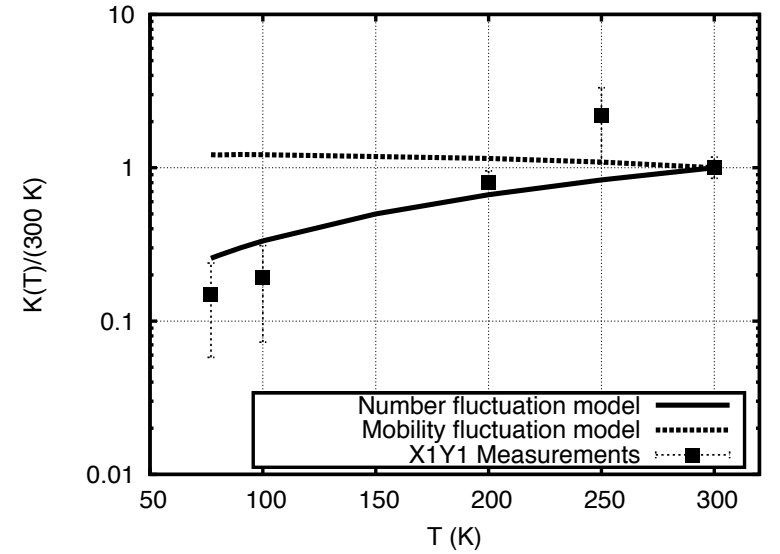

Fig. 4. Ratio $\mathrm{K}(\mathrm{T}) / \mathrm{K}(300 \mathrm{~K})$ with $\mathrm{K}$ defined by equation 1 as a function of temperature. An increase of the noise level is observed. The two solid lines in the figure are related to mobility fluctuations (equation 4) and to numbers fluctuations (equation 6). Only the number fluctuation assumption is able to account for the observed $1 /$ f noise level increase with the temperature.

\section{ACKNOWLEDGMENTS}

This work is supported by the EU under the Grant agreement N: PITN-GA-2008-213238, Initial training network RAINBOW of the 7 RTD Framework.

\section{REFERENCES}

[1] G. Pettinari, A. Polimeni, M. Capizzi, J. Blokland, P. Christianen, J. Maan, V. Lebedev, V. Cimalla, and O. Ambacher, "Carrier mass measurements in degenerate indium nitride," Physical Review B, vol. 79, no. 16, p. 165207, 2009.

[2] A. Bhuiyan, A. Hashimoto, and A. Yamamoto, "Indium nitride (inn): A review on growth, characterization, and properties," Journal of Applied Physics, vol. 94, p. 2779, 2003.

[3] K. Lin, J. Tsai, T. Wang, J. Hwang, M. Chen, and G. Chi, "Drift current dominated terahertz radiation from inn at low-density excitation," Applied Physics Letters, vol. 93, p. 262102, 2008.

4] V. Polyakov and F. Schwierz, "Low-field electron mobility in wurtzite inn," Applied Physics Letters, vol. 88, no. 3, p. 032101 , 2009 .

[5] F. Steranka, J. Bhat, D. Collins, L. Cook, M. Craford, R. Fletcher, N. Gardner, P. Grillot, W. Goetz, and M. Keuper, "High power leds-technology status and market applications," physica status solidi (a), vol. 194, no. 2, pp. 380-388, 2002.

[6] H. Lu, W. Schaff, L. Eastman, and C. Stutz, "Surface charge accumulation of inn films grown by molecular-beam epitaxy," Applied Physics Letters, vol. 82, p. 1736, 2003.

[7] J.-M. Routoure, D. Fadil, S. Flament, and L. Méchin, "A lownoise high output impedance dc current source," in 19th International Conference on Noise and Fluctuations; ICNF 200\%, vol. 922 , no. 1,2007 , pp. 419-424.

[8] J.-M. Routoure, B. Guillet, L. Méchin, A. Vilalta-Clemente, J. Grandal, M. Sánchez-García, F. Calle, and P. Ruterana, "Low frequency noise measurements in InN films," in Proceeding of EMRS 2009 Fall Meeting, Warsow, 2009.

[9] F. N. Hooge, "1/f noise is no surface effect," Physics Letters A, vol. 29 , no. 3 , pp. $139-140,1969$.

[10] F. Hooge, "Lattice scattering causes 1/f noise," Physics Letters $A$, vol. 66 , no. 4 , pp. 315-316, May 1978 .

[11] F. Hooge, T. Kleinpenning, and L. Vandamme, "Experimental studies on 1/f noise," Rep. Prog. Phys, vol. 44, 1981. 